Anaesthesist 2018 $\cdot 67: 380$

https://doi.org/10.1007/s00101-018-0426-5

Online publiziert: 26. März 2018

(c) Springer Medizin Verlag GmbH, ein Teil von Springer Nature 2018

CrossMark

R. Rossi

Herrieden, Deutschland

\title{
Ist ein supraglottischer Atemweg bei der Reanimation wirklich zu vermeiden?
}

men unterzogen wurden, die erwartungsgemäß nicht zum Erfolg führen können, unabhängig vom gewählten Ventilationsweg. Könnte also hier eine Umkehrung von Ursache und Wirkung vorliegen, also die Patienten mit der schlechten Prognose nur über supraglottische Atemwege beatmet werden und nicht, wie unterstellt, der Beatmungsweg deren schlechte Prognose bedingt?

Anders stellt es sich in den beiden anderen Gruppen dar.

In der Gruppe der primär (wohl häufig durch Notarzt) endotracheal Intubierten fallen Patienten mit von ihm zu erfassenden Hinweisen auf eine aussichtslose Situation heraus, und es kann bei indizierten Wiederbelebungsversuchen trotz geringer Bystander-CPR-Rate ein „besseres“ Ergebnis erzielt werden, was vielleicht unabhängig vom gewählten Beatmungshilfsmittel ist (?)

Die Gruppe SAD/ETI hat die höchste Beobachtungsrate, die höchste Bystander-CPR-Quote, den höchsten Anteil an Patienten mit schockbaren Rhythmen und schon deshalb die besten Aussichten auf eine erfolgreiche CPR. Womöglich völlig unabhängig vom Ventilationsweg? Sind nicht gerade hier die Patienten zu finden, bei denen das Team vor Ort eine Chance auf eine letztlich erfolgreiche Reanimation sieht und aufwendig weitertherapiert?

Dabei könnte auch die Regressionsanalyse mit anscheinend deutlich unterschiedlichem RACA Score durch „Hebeleffekte“ gleichgerichteter, also nicht unabhängiger Komponenten das Ergebnis Delta RACA gravierend beeinflussen.
Inwiefern wir auf der Basis der vorliegenden Daten der Hypothese der $\mathrm{Au}$ toren in der Zusammenfassung: „... die alleinige Anwendung von SGA vermieden und dass SGA in eine endotracheale Intubation überführt werden sollten “ folgen und angesichts des (Zeit-)Aufwands und der mit einer Umintubation verbundenen Risiken unser Vorgehen umstellen sollten, könnte bezweifelt werden. Auch die Auswirkungen derartiger Hypothesen auf das Vorgehen der (zukünftigen) Notfallsanitäter muss mitbeachtet werden.

\section{Korrespondenzadresse}

\section{Dr. R. Rossi}

Herrieden, Deutschland

rolando.rossi@web.de

Interessenkonflikt. R. Rossi gibt an, dass kein Interessenkonflikt besteht. 